\title{
A Single-Exponential Time 2-Approximation Algorithm for Treewidth
}

\author{
Tuukka Korhonen*
}

June 4, 2021

\begin{abstract}
We give an algorithm, that given an $n$-vertex graph $G$ and an integer $k$, in time $2^{O(k)} n$ either outputs a tree decomposition of $G$ of width at most $2 k+1$ or determines that the treewidth of $G$ is larger than $k$. This is the first 2-approximation algorithm for treewidth that is faster than the known exact algorithms. In particular, our algorithm improves upon both the previous best approximation ratio of 5 in time $2^{O(k)} n$ and the previous best approximation ratio of 3 in time $2^{O(k)} n^{O(1)}$, both given by Bodlaender et al. [FOCS 2013, SICOMP 2016]. Our algorithm is based on a local improvement method adapted from a proof of Bellenbaum and Diestel [Comb. Probab. Comput. 2002].
\end{abstract}

${ }^{*}$ Department of Computer Science, University of Helsinki, Finland. Email: tuukka.m.korhonen@helsinki.fi. Homepage: https://tuukkakorhonen.com. 


\section{Introduction}

Treewidth is one of the most important graph parameters, playing a central role in multiple fields. In particular, many graph problems that are intractable in general can be solved in time $f(k) n^{O(1)}$ when the input includes also a tree decomposition of width $k$ [Cou90]. For a large number of classical problems there are in fact such algorithms with time complexity $2^{O(k)} n$ [AP89, Bod88, BCKN15]. To use these algorithms, it is crucial to also have an algorithm for finding a tree decomposition with near-optimal width. In particular, in order to truly obtain algorithms with time complexity $2^{O(k)} n$ for these problems, where $k$ is the treewidth of the input graph, we need to be able to compute a tree decomposition of width $c k$, for some constant $c$, in time $2^{O(k)} n$. Moreover, lowering the constant $c$ directly speeds up all of these algorithms.

There is a long history of algorithms for finding tree decompositions with different kinds of guarantees on the width of the decomposition and on the running time of the algorithm. See Table 1 for an overview of the most relevant of these results. The first constant-factor approximation algorithm with running time of type $f(k) n^{O(1)}$ was given by Robertson and Seymour in their graph minors series [RS95]. The dependency on $n$ in this algorithm was improved to $n \log n$ by Reed [Ree92], with the cost of a worse approximation ratio and dependency on $k$. Bodlaender introduced the first algorithm for treewidth with a linear dependency on $n$ [Bod93, Bod96]. The algorithm of Bodlaender in fact computes a tree decomposition of optimal width, but with a running time dependency of $2^{O\left(k^{3}\right)}$ on the width $k$.

While after the first half of the 1990s multiple improvements to treewidth approximation were given [FHL08, Ami10], the problem of constant-factor approximating treewidth in $2^{O(k)} n$ time stood until 2013, when Bodlaender, Drange, Dregi, Fomin, Lokshtanov, and Pilipczuk gave a $2^{O(k)} n$ time 5 -approximation algorithm for treewidth $\left[\mathrm{BDD}^{+} 13, \mathrm{BDD}^{+} 16\right]$. The same authors also gave a 3 -approximation algorithm with running time $2^{O(k)} n \log n$. The 3 -approximation algorithm of Bodlaender et al. has currently the best approximation ratio achieved by other than the exact algorithms.

Table 1: Overview of treewidth algorithms with time complexity $f(k) \cdot g(n)$, each either outputting a tree decomposition of width at most $\alpha(k)$ or determining that the treewidth of the input graph is larger than $k$. Most of the rows are based on a similar table in $\left[\mathrm{BDD}^{+} 16\right]$.

\begin{tabular}{|c|c|c|c|}
\hline Reference & Approximation $\alpha(k)$ & $f(k)$ & $g(n)$ \\
\hline Arnborg, Corneil, and Proskurowski [ACP87] & exact & $O(1)$ & $n^{k+2}$ \\
Robertson and Seymour [RS95] & $4 k+3$ & $O\left(3^{3 k}\right)$ & $n^{2}$ \\
Lagergren [Lag96] & $8 k+7$ & $2^{O(k \log k)}$ & $n \log ^{2} n$ \\
Reed [Ree92] & $8 k+O(1)$ & $2^{O(k \log k)}$ & $n \log n$ \\
Bodlaender [Bod96] & exact & $2^{O\left(k^{3}\right)}$ & $n$ \\
Amir [Ami10] & $4.5 k$ & $O\left(2^{3 k} k^{3 / 2}\right)$ & $n^{2}$ \\
Amir [Ami10] & $(3+2 / 3) k$ & $O\left(2^{3.6982 k} k^{3}\right)$ & $n^{2}$ \\
Amir [Ami10] & $O(k \log k)$ & $O(k \log k)$ & $n^{4}$ \\
Feige, Hajiaghayi, and Lee [FHL08] & $O(k \sqrt{\log k})$ & $O(1)$ & $n$ \\
Fomin, Todinca, and Villanger [FTV15] & exact & $O(1)$ & $1.7347^{n}$ \\
Fomin et al. [FLS 18$]$ & $O\left(k^{2}\right)$ & $O\left(k^{7}\right)$ & $n \log n$ \\
Bodlaender et al. [BDD ${ }^{+}$16] & $3 k+4$ & $2^{O(k)}$ & $n \log n$ \\
Bodlaender et al. [BDD ${ }^{+}$16] & $5 k+4$ & $2^{O(k)}$ & $n$ \\
This paper & $2 k+1$ & $2^{O(k)}$ & $n$ \\
\hline
\end{tabular}


In this paper we improve upon both of the algorithms given in $\left[\mathrm{BDD}^{+} 16\right]$.

Theorem 1.1. There is an algorithm, that given an $n$-vertex graph $G$ and an integer $k$, in time $2^{O(k)} n$ either outputs a tree decomposition of $G$ of width at most $2 k+1$ or determines that the treewidth of $G$ is larger than $k$.

To further compare our algorithm to the results of Bodlaender et al., we remark that our algorithm has significantly smaller exponential dependency on $k$ hidden in the $2^{O(k)}$ factor than what the techniques of $\left[\mathrm{BDD}^{+} 16\right]$ yield, although we note that the main goal of neither our work nor their work was to optimize this factor. Our algorithm also makes progress in that it is the first treewidth approximation algorithm to significantly deviate from the basic shape introduced by Robertson and Seymour [RS95]. In particular, all of the previous treewidth approximation algorithms [RS95, Lag96, Ree92, Ami10, FHL08, $\left.\mathrm{BDD}^{+} 16, \mathrm{FLS}^{+} 18\right]$ follow the same basic structure of recursively finding a separator that splits the previously found separator (and in some cases also the component) in a balanced manner. Our algorithm is instead based on iteratively making local improvements to a tree decomposition, with the method of improvement based on the work of Bellenbaum and Diestel [BD02]. To the best of my knowledge, our algorithm is the first to apply the technique of Bellenbaum and Diestel in the context of computing treewidth. The technique of Bellenbaum and Diestel has been applied before $\left[\mathrm{CKL}^{+} 21\right.$, LSS20] for optimizing a different criterion on tree decompositions, with applications to improved parameterized algorithms for minimum bisection, Steiner cut, and Steiner multicut [CKL $\left.{ }^{+} 21\right]$, and for obtaining a parameterized approximation scheme for minimum $k$-cut [LSS20].

Before describing our algorithm we further mention some related work. There are multiple refinements of the $2^{O\left(k^{3}\right)} n$ time exact treewidth algorithm of Bodlaender [Bod96], including in particular the version of Perkovic and Reed [PR00] that has applications to the disjoint paths problem and the logarithmic space version of Elberfeld, Jakoby, and Tantau [EJT10]. Also, recently Bodlaender, Jaffke, and Telle gave additional structural insights on the technique of typical sequences used in the algorithm [BJT21]. While exact computing of treewidth is known to be NPcomplete [ACP87], currently the strongest hardness result against constant-factor approximation is that by $\mathrm{Wu}$, Austrin, Pitassi, and Liu, assuming the small set expansion conjecture, there is no polynomial-time $c$-approximation algorithm for treewidth for any constant $c$ [WAPL14]. Recently, Groenland, Joret, Nadara, and Walczak gave a polynomial-time $(k+1)$-approximation algorithm for pathwidth, where $k$ is the width of a tree decomposition given as an input [GJNW21].

\subsection{Outline of The Algorithm}

Our algorithm is based on a proof of Bellenbaum and Diestel, in particular, on the proof of Theorem 3 in [BD02]. The proof of Bellenbaum and Diestel shows that if a tree decomposition has a bag that is not "lean", ${ }^{1}$ then this bag can be split in a manner that improves the tree decomposition. In particular, if we split a bag of size $w+1$ in a tree decomposition of width $w$, then the splitting does not increase the width of the decomposition and decreases the number of bags of size $w+1$.

It follows that when starting with an initial tree decomposition of width $O(k)$, we can obtain a tree decomposition whose largest bag is lean by applying $O(n k)$ splitting operations, each of which can be implemented in $2^{O(k)} n^{O(1)}$ time. A lean vertex set has at most $3 k+3$ vertices [HW17], where $k$ is the treewidth, so this directly gives a $2^{O(k)} n^{O(1)}$ time 3 -approximation algorithm for treewidth. However, for each $k$ there are graphs that have lean vertex sets of size $3 k-3$, so this technique does not directly give a better approximation ratio than 3 .

\footnotetext{
${ }^{1}$ We omit the definition of "lean" because it is ultimately not used in our algorithm.
} 


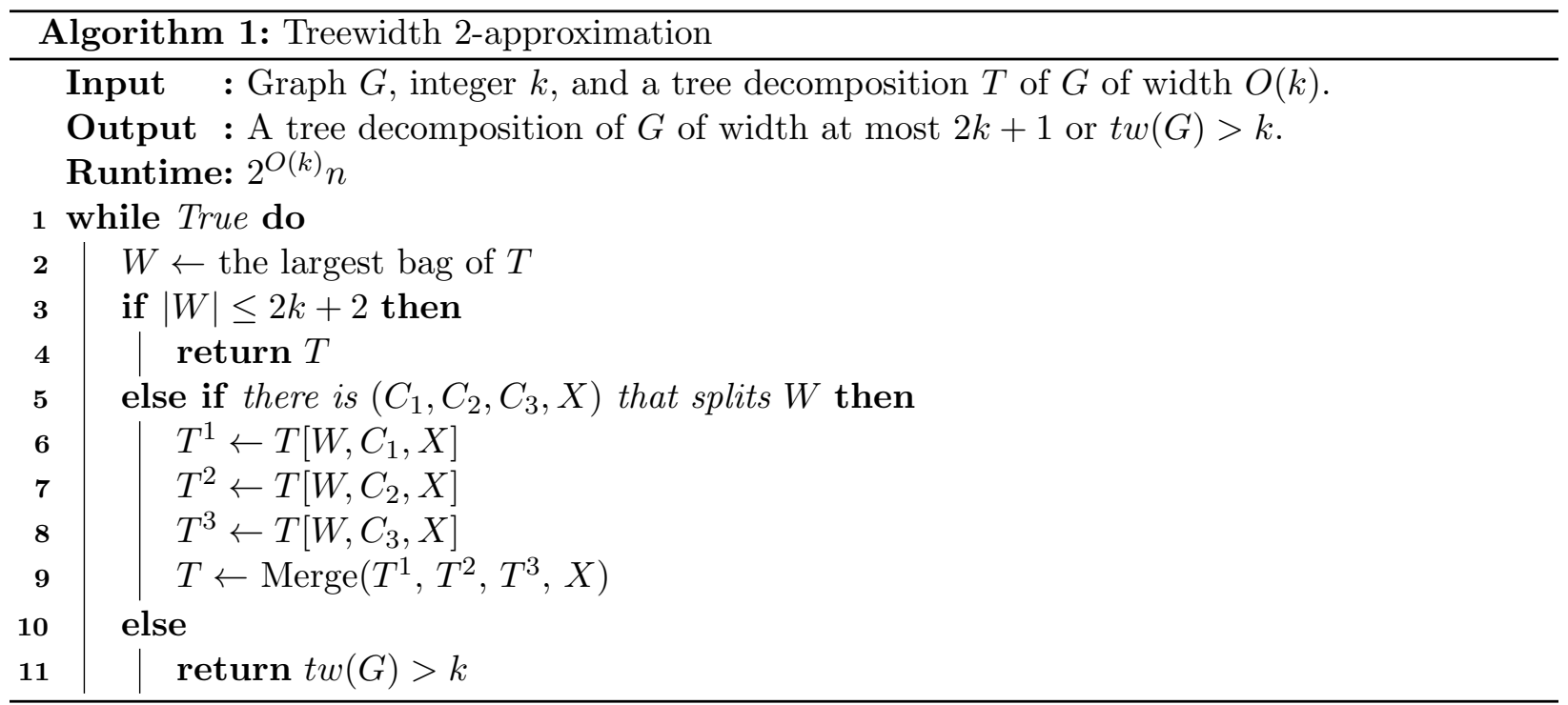

To improve the approximation ratio, we show that if we generalize the bag splitting operation from 2 -way splits to 3 -way splits, then we can improve the tree decomposition by splitting the largest bag as long as its size is larger than $2 k+2$. In particular, we say that a bag $W$ is splittable if the vertices of the graph can be partitioned into $\left(C_{1}, C_{2}, C_{3}, X\right)$ so that $\left|\left(C_{i} \cap W\right) \cup X\right|<|W|$ for each $i$ and there are no edges between $C_{i}$ and $C_{j}$ for $i \neq j$. Algorithm 1 gives a high-level presentation of the algorithm as pseudocode.

A straightforward implementation of the algorithm with standard techniques yields time complexity $2^{O(k)} n^{O(1)}$. To optimize the dependency on $n$ to be linear, we first show that the improvements to the tree decomposition can be implemented so that over the course of the algorithm, in total $2^{O(k)} n$ bag edits are made, and the bag edits of each improvement step are limited to a connected subtree around the split bag $W$. We implement computing the splits by dynamic programming over the tree decomposition that we are also editing at the same time.

Our algorithm depends on having an initial tree decomposition of width $O(k)$ as an input. By the compression technique of Bodlaender [Bod96] (Lemma 2.7 in $\left[\mathrm{BDD}^{+} 16\right]$ ), any approximation algorithm for treewidth that outputs a tree decomposition of width $\alpha(k)$ can be assumed to have a tree decomposition of width at most $2 \alpha(k)+1$ as an input, incurring an overhead of factor $k^{O(1)}$ to the running time. In particular, in our algorithm we can assume a tree decomposition of width $4 k+3$ as an input. Our algorithm does not depend on black-box use of any other results than this compression technique.

\section{Preliminaries}

\section{$2.1 \quad$ Notation}

We use the convention that a partition of a set may contain empty parts.

The vertices of a graph $G$ are denoted by $V(G)$ and edges by $E(G)$. The subgraph induced by a vertex set $X \subseteq V(G)$ is denoted by $G[X]$ and the subgraph induced by a vertex set $V(G) \backslash X$ is denoted by $G \backslash X$.

We treat paths as sequences of vertices and all paths in this paper are required to be simple, i.e., have no repetitions. Let $X$ and $Y$ be possibly overlapping vertex sets. A vertex set $S$ separates 
$X$ from $Y$ if any path that intersects both $X$ and $Y$ intersects also $S$.

A tree is a connected acyclic graph. A subtree is a connected induced subgraph of a tree. To distinguish trees from graphs, vertices of a tree are called nodes. A rooted tree has one node $r$ chosen as a root. A node $j$ of a rooted tree is a descendant of a node $i$ if $i$ separates $j$ from $r$. Conversely, such $i$ is an ancestor of such $j$. If $i \neq j$ the ancestor/descendant relation may be called strict and if $i$ and $j$ are adjacent they may be called parent/child. A rooted subtree of a rooted tree is a subtree that consists of all descendants of some node.

\subsection{Tree Decompositions}

A tree decomposition of a graph $G$ is a tree $T$ whose each node $i \in V(T)$ is associated with a bag $B_{i} \subseteq V(G)$ such that

1. $V(G)=\bigcup_{i \in V(T)} B_{i}$,

2. for each $\{u, v\} \in E(G)$ there is $B_{i}$ with $\{u, v\} \subseteq B_{i}$, and

3. for each $v \in V(G)$ the set of nodes $\left\{i \in V(T) \mid v \in B_{i}\right\}$ forms a subtree of $T$.

The width of a tree decomposition is $\max _{i \in V(T)}\left|B_{i}\right|-1$. We will often abuse notation by talking about a bag $B_{i}$ instead of the node $i$. In our algorithm we usually treat a tree decomposition $T$ as rooted on some selected root node, whose bag is usually denoted with $W$. With respect to a root bag $W$, the home bag $B_{x}$ of a vertex $x \in V(G)$ is the bag with $x \in B_{x}$ that is the closest to $W$ in $T$. Note that all bags containing $x$ are descendants of $B_{x}$ in $T$.

We will use the following standard lemma implicitly throughout the paper.

Lemma 2.1. Let $T_{1}, T_{2}$ be subtrees of a tree decomposition $T$ of a graph $G$ that are separated by a node $i$ of $T$. The vertex sets $V_{1}=\bigcup_{j \in V\left(T_{1}\right)} B_{j}$ and $V_{2}=\bigcup_{j \in V\left(T_{2}\right)} B_{j}$ are separated by $B_{i}$ in $G$.

Let $W \subseteq V(G)$ be any vertex set of $G$. A balanced separator of $W$ is a vertex set $X$ such that for each connected component $C_{i}$ of $G \backslash X$ it holds that $\left|W \cap C_{i}\right| \leq|W| / 2$. The existence of balanced separators with size bounded by treewidth is a classical lemma from Graph Minors II.

Lemma 2.2 ([RS86]). Let $G$ be a graph of treewidth $k$ and $W \subseteq V(G)$ a vertex set of $G$. There is a balanced separator $X$ of $W$ of size $|X| \leq k+1$.

\subsection{Bodlaender's Compression Technique}

The only black-box result that our algorithm relies on is the compression technique of Bodlaender, introduced in [Bod96] and also exploited in $\left[\mathrm{BDD}^{+} 16\right]$. Now we briefly discuss on how we use the technique. For more details on the technique we refer to [Bod96] and on its application to treewidth approximation to $\left[\mathrm{BDD}^{+} 16\right]$.

Proposition 2.3 ([Bod96]). There is an algorithm, that given an n-vertex graph $G$ and an integer $k$, in $k^{O(1)} n$ time either

1. determines that the treewidth of $G$ is larger than $k$,

2. returns a matching in $G$ with at least $n / O\left(k^{6}\right)$ edges, or

3. returns a graph $G^{\prime}$ with at most $n-n / O\left(k^{6}\right)$ vertices so that the treewidth of $G^{\prime}$ is at most the treewidth of $G$ if the treewidth of $G$ at most $k$, and furthermore, any tree decomposition of $G^{\prime}$ of width $\leq k$ can be turned into a tree decomposition of $G$ of width $\leq k$ in $k^{O(1)} n$ time. 
In particular, in our case Proposition 2.3 is exploited in the following way (which is very similar to $\left[\mathrm{BDD}^{+} 16\right]$; we include a proof for the convenience of the reader).

Lemma 2.4. Suppose there is an algorithm $A$, that given an n-vertex graph $G$, integer $k$, and $a$ tree decomposition of $G$ of width at most $4 k+3$, in time $f(k) n$ either outputs a tree decomposition of $G$ of width at most $2 k+1$ or determines that the treewidth of $G$ is larger than $k$. Then there is an algorithm that in time $f(k) k^{O(1)} n$ does the same, but without requiring a tree decomposition as an input.

Proof. We use a recursive procedure, which is always called with parameters $G$ and $k$, where $k$ is the original input $k$ and $G$ is a graph whose treewidth is at most $k$ if the treewidth of the input graph is at most $k$. Each recursive call either determines that the treewidth of $G$ (and thus also the treewidth of the input graph) is larger than $k$, or returns a tree decomposition of $G$ of width at most $2 k+1$.

The base case of the recursion is a trivial edgeless graph with treewidth 0 . In the start of each recursive call we use the algorithm of Proposition 2.3 with parameters $(G, 2 k+1)$. In case 1 , we can return immediately. In case 3 , we call the procedure recursively with the graph $G^{\prime}$, and in case of a positive answer construct the tree decomposition of $G$ of width at most $2 k+1$ and return it. In case 2 , we contract the edges of the matching to obtain a graph $G_{M}$ and call the algorithm recursively on $G_{M}$. Contracting edges does not increase treewidth, so the treewidth of $G_{M}$ is at most the treewidth of $G$. Also, in particular, we can obtain a tree decomposition $T$ of $G$ of width $4 k+3$ from a tree decomposition of $G_{M}$ of width $2 k+1$ by expanding the bags according to the matching. Then we use the algorithm $A$ with $T$ to either get a tree decomposition of width $2 k+1$ or to determine that the treewidth of $G$ is larger than $k$.

In each recursive call the number of vertices of $G$ shrinks by a factor $1 / O\left(k^{6}\right)$, and therefore the total time complexity is $T(n, k)=k^{O(1)} n+f(k) n+T\left(n-n / O\left(k^{6}\right), k\right)$, which can be bounded by $T(n, k)=f(k) k^{O(1)} n$.

\section{Main Concepts of The Algorithm}

In this section we give a description of our algorithm and prove its correctness, but do not optimize the time complexity. In particular, after this section it should be clear that we have a 2 -approximation algorithm that can be implemented in time $2^{O(k)} n^{O(1)}$. In Section 4 we optimize the dependency on $n$ to be linear.

Many proof ideas of this section are based on [BD02], but all of the proofs have been generalized or adapted in some ways.

\subsection{Splittable Vertex Sets}

A vertex set $W \subseteq V(G)$ is splittable if $V(G)$ can be partitioned into $\left(C_{1}, C_{2}, C_{3}, X\right)$ such that there are no edges between $C_{i}$ and $C_{j}$ for $i \neq j$ and $\left|\left(W \cap C_{i}\right) \cup X\right|<|W|$ for all $i$. We refer to such 4-tuple as a split of $W$.

Lemma 3.1. Let $G$ be a graph of treewidth $\leq k$. Any vertex set $W \subseteq V(G)$ of size $|W| \geq 2 k+3$ is splittable.

Proof. By Lemma 2.2, there is a balanced separator $X$ of $W$ of size $|X| \leq k+1$. Now, as each connected component $C_{i}$ of $G \backslash X$ has $\left|C_{i} \cap W\right| \leq|W| / 2$, we can combine two such components with the smallest sizes of $C_{i} \cap W$ until we obtain a partition $\left(C_{1}, C_{2}, C_{3}, X\right)$ of $V(G)$, where it holds 
that $\left|W \cap C_{i}\right| \leq|W| / 2$, and there are no edges between $C_{i}$ and $C_{j}$ for distinct $i, j$. This is indeed a split of $W$ because $\left|\left(W \cap C_{i}\right) \cup X\right| \leq|W| / 2+k+1<|W|$.

Let $W$ be a root bag of a tree decomposition $T$. A split $\left(C_{1}, C_{2}, C_{3}, X\right)$ is a minimum split of $W$ if the split minimizes $|X|$ among all splits of $W$, and among splits minimizing $|X|$ the split minimizes $d(X)=\sum_{x \in X} d(x)$, where $d(x)$ is the distance from the home bag $B_{x}$ of $x$ to $W$ in $T$.

\subsection{The Splitting Operation}

Let $T$ be a tree decomposition, $W$ a root bag of $T$, and $\left(C_{1}, C_{2}, C_{3}, X\right)$ a minimum split of $W$. We obtain a tree decomposition $T\left[W, C_{i}, X\right]$ of $G\left[C_{i} \cup X\right]$ by removing all other vertices than $\left(C_{i} \cup X\right)$ from each bag of $T$, and inserting each vertex $x \in X$ to all bags in the path from the root $W$ to the home bag $B_{x}$ of $x$. In other words, for each bag $B$ of $T$, we have a bag $B^{i}=\left(B \cap\left(C_{i} \cup X\right)\right) \cup B^{X}$ in $T\left[W, C_{i}, X\right]$, where the set $B^{X}$ is defined as $B^{X}=\left\{x \in(X \backslash B) \mid B_{x}\right.$ is a descendant of $B$ in $\left.T\right\}$. Note that the insertions $B^{X}$ can be seen as first adding $X$ to the root bag, and them "fixing" the subtree condition by adding vertices $x \in X$ to bags in a minimal way.

Now $T\left[W, C_{i}, X\right]$ is a tree decomposition of $G\left[C_{i} \cup X\right]$ because we removed only vertices not in $C_{i} \cup X$ from bags and we inserted vertices $x \in X$ to paths touching $B_{x}$, maintaining the subtree condition. Note also that the root bag $W^{i}$ of $T\left[W, C_{i}, X\right]$ has $X \subseteq W^{i}$, so the tree decompositions $T\left[W, C_{1}, X\right], T\left[W, C_{2}, X\right]$, and $T\left[W, C_{3}, X\right]$ can be merged into a tree decomposition $T^{\prime}$ of $G$ by adding an additional bag $X$ connected to each $W^{i}$. We refer to $T^{\prime}$ as the improved tree decomposition.

The following Lemma gives the main argument to show that the improved tree decomposition is indeed improved.

Lemma 3.2. Consider the construction given above and let $B$ be any bag of T. If $B^{X}$ is non-empty then $\left|B^{X}\right|<\left|B \cap\left(C_{i} \cup C_{j}\right)\right|$ for any pair of distinct $i, j$.

Proof. For simplicity w.l.o.g. let $i=1, j=2$. Suppose that $\left|B^{X}\right| \geq\left|B \cap\left(C_{1} \cup C_{2}\right)\right|$. We claim that there is a split $\left(C_{1}^{\prime}, C_{2}^{\prime}, C_{3}^{\prime}, X^{\prime}\right)$ of $W$ with $X^{\prime}=\left(X \backslash B^{X}\right) \cup\left(B \cap\left(C_{1} \cup C_{2}\right)\right)$. This split would contradict the minimality of the original split because $\left|X^{\prime}\right| \leq|X|$ and the home bags of vertices in $B^{X}$ are strict descendants of $B$ and thus strict descendants of the home bags of vertices in $B \cap\left(C_{1} \cup C_{2}\right)$, implying $d(y)<d(x)$ for all $y \in B \cap\left(C_{1} \cup C_{2}\right)$ and $x \in B^{X}$.

To show that there is indeed such a split $\left(C_{1}^{\prime}, C_{2}^{\prime}, C_{3}^{\prime}, X^{\prime}\right)$, first note that $B^{X}$ does not intersect $W$ because $B$ separates it from $W$ and $B \cap B^{X}=\emptyset$, so $W \cap X \subseteq W \cap X^{\prime}$. Next we prove that the vertex sets $\left(W \cap C_{1}\right) \backslash X^{\prime},\left(W \cap C_{2}\right) \backslash X^{\prime}$, and $\left(W \cap C_{3}\right) \backslash X^{\prime}$ are in different components of $G \backslash X^{\prime}$. Suppose that there is a path from $\left(W \cap C_{k}\right) \backslash X^{\prime}$ to $\left(W \cap C_{l}\right) \backslash X^{\prime}$, with $k \neq l$, in $G \backslash X^{\prime}$, and by symmetry assume that $k \in\{1,2\}$. The path must intersect $B^{X}$ before intersecting other vertices of $V(G) \backslash C_{k}$ because $X^{\prime} \cup B^{X} \supseteq X$ separates $W \cap C_{k}$ from $V(G) \backslash C_{k}$. Therefore we have a path from $W \cap C_{k}$ to $B^{X}$ that is contained in $\left(C_{k} \cup B^{X}\right) \backslash X^{\prime}$. This path must have a vertex in $B$ because $B$ separates $W$ from $B^{X}$. However $B \cap\left(C_{k} \cup B^{X}\right)=B \cap C_{k} \subseteq X^{\prime}$, so this path cannot have a vertex in $B$.

It follows that $\left|B^{i}\right| \leq|B|$ for all $B^{i}$, and that $\left|B^{i}\right|<|B|$ if $B$ intersects at least two of $C_{1}, C_{2}, C_{3}$. This shows that the width of the improved tree decomposition $T^{\prime}$ is at most the width of $T$, and that the number of bags of size $|W|$ in $T^{\prime}$ is smaller than in $T$ if $W$ is a largest bag of $T$. In particular, note that by the definition of a split we that $\left|W^{i}\right|<|W|$ for the root bag $W$ and all $i$, and by Lemma 3.2 the only case when $\left|B^{i}\right|=|B|$ can hold for multiple $i$ is when $B \subseteq X$. 
We note that already at this point, our arguments coupled with applying standard techniques to compute minimum splits in time $2^{O(k)} n^{O(1)}$ lead to an $2^{O(k)} n^{O(1)}$ time 2-approximation algorithm for treewidth. What remains is to optimize the dependency on $n$.

\subsection{Local Splitting}

We define the potential function $\phi$ on a tree decomposition $T$ as a sum $\phi(T)=\sum_{i \in V(T)} 7^{\left|B_{i}\right|}$ over the bags $B_{i}$ of $T$. In this subsection we will give a slightly modified splitting operation for which it will hold that $\phi\left(T^{\prime \prime}\right)<\phi(T)$, where $T^{\prime \prime}$ is the tree decomposition obtained by splitting a tree decomposition $T$, and this splitting operation can be implemented by modifying a subtree of $T$ consisting of $O\left(\phi(T)-\phi\left(T^{\prime \prime}\right)\right)$ nodes and containing $W$.

We say that a bag $B$ of $T$ is editable with respect to a split $\left(C_{1}, C_{2}, C_{3}, X\right)$ of the root bag $W$ if $B$ intersects at least two of $C_{1}, C_{2}, C_{3}$ and each bag in the path in $T$ from $B$ to $W$ is editable. The root bag $W$ is editable in any split of $W$, so the editable bags form a non-empty subtree of $T$ containing $W$. Note also that because the root $W$ is in the subtree of editable bags, the noneditable bags form a collection of rooted subtrees of $T$. It follows from Lemma 3.2 that if $B$ is a non-editable bag in a minimum split, then $B^{X}=\emptyset$.

Next we provide a construction of the tree decomposition $T^{\prime \prime}$ similar to the construction of $T^{\prime}$ in Section 3.2. The tree decomposition $T^{\prime \prime}$ is essentially the same as $T^{\prime}$, except that some components of $G \backslash X$ that do not intersect $W$ have been re-assigned to a different part $C_{i}$ of the split, and some rooted subtrees whose bags contain only vertices in $X$ have been pruned. This allows to construct $T^{\prime \prime}$ by only modifying the subtree of editable bags of $T$. We also maintain an invariant that the maximum degree of the tree decomposition is at most 3 .

Lemma 3.3. Let $T$ be a degree-3 tree decomposition of a graph $G$ of width $w$ with a root bag $W$ with $|W|=w+1,\left(C_{1}, C_{2}, C_{3}, X\right)$ a minimum split of $W$, and the number of editable bags of $T$ with respect to the split. We can obtain a degree-3 tree decomposition $T^{\prime \prime}$ of $G$ by removing the editable bags from $T$ and inserting at most $3 t+4$ bags in place of them. Moreover, $\phi\left(T^{\prime \prime}\right) \leq \phi(T)-t$ and all of the new bags inserted have size $<|W|$.

Proof. We start the construction of $T^{\prime \prime}$ similarly as $T^{\prime}$ in Section 3.2, but limited only to editable bags. In particular, for each editable bag $B$ of $T$ we have bags $B^{i}=\left(B \cap\left(C_{i} \cup X\right)\right) \cup B^{X}$ for $i \in\{1,2,3\}$ in $T^{\prime \prime}$. Then we add a bag $X$ and connect the bags $W^{i}$ to $X$. The nodes of bags $W^{i}$ may become degree- 4 here but we will handle that later.

The non-editable bags of $T$ form at most $3 t$ rooted subtrees of $T$. We remove the editable bags of $T$ and connect the non-editable subtrees of $T$ to $T^{\prime \prime}$. In particular, let $B$ be a non-editable bag of $T$ whose parent bag $P$ is editable. The bag $B$ intersects at most one component $C_{i}$. We connect $B$ to $P^{i}$ such that $B \subseteq C_{i} \cup X$. Note that the rooted subtree of non-editable bags rooted at $B$ may contain bags that contain vertices that have been assigned to some other components $C_{j}, j \neq i$, of the split. However, $B$ separates all vertices in the subtree of $B$ from $W$ and $B \subseteq C_{i} \cup X$, so we can assign all vertices in the subtree of $B$ that are not in $X$ to $C_{i}$ without affecting $C_{k} \cap W$ for any $k$. With this re-assignment, the subtree of $B$ is the same as the subtree of $B^{i}$ would be in the original construction $T^{\prime}$. Now it suffices to show that it is safe to not include the subtrees of $B^{j}$, $j \neq i$ at all in $T^{\prime \prime}$. Such subtrees would contain only vertices of $X$, so they can be pruned because we anyway have a bag $X$ in $T^{\prime \prime}$.

Some $W^{i}$ may have become degree-4, in particular, it might have children $W_{a}^{i}, W_{b}^{i}, W_{c}^{i}$ and parent $X$. In this case we add a new bag $W_{d}^{i}=W^{i}$ connected to $W^{i}, W_{a}^{i}$, and $W_{b}^{i}$, and remove the edges between $W^{i}$ and $W_{a}^{i}, W_{b}^{i}$. The degree of any other node does not change, so applying this to each $W^{i}$ makes $T^{\prime \prime}$ degree-3. 
Let $E$ be the set of editable bags of $T$. By Lemma 3.2 we have for $B \in E$ that $\left|B^{i}\right|=$ $|B|-\left|B \cap\left(C_{j} \cup C_{k}\right)\right|+\left|B^{X}\right|<|B|$, where $i, j, k$ are distinct. We also have that $|B| \geq 2$ for all $B \in E$ and $|X| \leq|W|-2$. Let $E^{\prime}=E \backslash\{W\}$. The potential of $T^{\prime \prime}$ is

$$
\begin{aligned}
\phi\left(T^{\prime \prime}\right) & \leq \phi(T)-7^{|W|}-\left(\sum_{B \in E^{\prime}} 7^{|B|}\right)+7^{|X|}+\sum_{i \in\{1,2,3\}}\left(7^{\left|W^{i}\right|}+7^{\left|W_{d}^{i}\right|}+\sum_{B \in E^{\prime}} 7^{\left|B^{i}\right|}\right) \\
& \leq \phi(T)+6 \cdot 7^{|W|-1}-7^{|W|}+7^{|X|}+\sum_{B \in E^{\prime}}\left(3 \cdot 7^{|B|-1}-7^{|B|}\right) \\
& \leq \phi(T)-7^{|W|-1}+7^{|W|-2}-4(t-1) \leq \phi(T)-t .
\end{aligned}
$$

Because the value of the potential function of the initial tree decomposition is $2^{O(k)} n$, our algorithm will terminate after editing $2^{O(k)} n$ bags.

\section{Implementation in Linear Time}

In this section we show that our algorithm can be implemented in $2^{O(k)} n$ time. In particular, this requires showing that finding a split can be done in amortized $2^{O(k)}$ time and that the local splitting of Section 3.3 can be implemented in $2^{O(k)} t$ time, where $t$ is the number of editable bags.

\subsection{Overview}

We treat our algorithm in the form that we are given a graph $G$, an integer $k$, and a degree-3 tree decomposition $T$ of $G$ of width $w$, where $2 k+2 \leq w \leq 4 k+3$, where the upper bound is by Lemma 2.4. The algorithm either outputs a tree decomposition of width at most $w-1$, or concludes that the treewidth of $G$ is larger than $k$. Note that given a tree decomposition $T$ of width $w$, we can obtain a tree decomposition of maximum degree 3 , width $w$, and $O(n)$ bags in $w^{O(1)}(n+|T|)$ time by standard techniques [Klo94].

During the algorithm we maintain a degree-3 tree decomposition $T$ and a pointer to a node $r$ of $T$. We treat $T$ as rooted on node $r$ and we denote by $W$ the bag of $r$. We implement a data structure that supports the following operations.

1. Init $(T, r)$ - Initializes the data structure with a degree-3 tree decomposition $T$ of width $w$ and a node $r \in V(T)$ in time $2^{O(w)} n$.

2. Move $(s)$ - Moves the pointer from $r$ to an adjacent node $s$ in time $2^{O(w)}$.

3. Split () - Returns $\perp$ if the bag $W$ of $r$ is not splittable, otherwise sets the internal state to a minimum split $\left(C_{1}, C_{2}, C_{3}, X\right)$ of $W$ and returns $T$. Works in $2^{O(w)}$ time.

4. State () - Returns the internal state of the data structure restricted to the bag $W$ of $r$, i.e., the partition $\left(C_{1} \cap W, C_{2} \cap W, C_{3} \cap W, X \cap W\right)$ of $W$. Works in $w^{O(1)}$ time. Valid only if there has been a successful Split query after the previous Init or Edit query.

5. $\operatorname{Edit}\left(T_{1}, T_{2}, p, r^{\prime}\right)$ - Replaces a subtree $T_{1}$ of $T$ with a given subtree $T_{2}$, where $r \in V\left(T_{1}\right)$, $r^{\prime} \in V\left(T_{2}\right)$, and $p$ is a function from the nodes of $T \backslash T_{1}$ whose parents are in $T_{1}$ to the nodes of $T_{2}$, specifying how $T \backslash T_{1}$ will be connected to $T_{2}$. The pointer $r$ will be set to $r^{\prime}$. Works in $2^{O(w)}\left(\left|T_{1}\right|+\left|T_{2}\right|\right)$ time. Assumes the new $T$ to have degree-3 and width at most $w$.

We defer the description on how the operations are implemented to Section 4.3. 


\subsection{Splitting With The Data Structure}

We describe how the data structure is used to implement the local splitting of Section 3.3 to split all bags of size $w+1$ in a tree decomposition of width $w$ in time $2^{O(w)} n$, or to report that there is a bag of size $w+1$ that cannot be split.

We traverse the tree decomposition in depth-first order with the Move operations. Each time we reach a bag $W$ of size $|W|=w+1$, we apply the Split operation, returning $t w(G)>k$ if it returns $\perp$. If the Split operation returns $T$, we use the Move and State operations to explore the subtree of editable bags and the non-editable children of them. As our tree decomposition has maximum degree 3 , the number of non-editable children of $t$ editable bags is bounded by $3 t$. We determine the sets $B^{X}$ for each editable bag $B$ recursively, in particular noting that $B^{X}=\emptyset$ for non-editable bags by Lemma 3.2 , and that if $B_{1}, B_{2}$, and $B_{3}$ are the children of $B$, then $B^{X}=B_{1}^{X} \cup B_{2}^{X} \cup B_{3}^{X} \cup\left(X \cap\left(B_{1} \cup B_{2} \cup B_{3}\right) \backslash B\right)$. Then we can implement the construction of Lemma 3.3 with the Edit operation in $2^{O(w)} t$ time, where $t$ is the number of editable bags.

Now what is left is to show that the depth-first search visiting all nodes can indeed be implemented with $2^{O(w)} n$ Move operations despite the edits to the tree decomposition during the search. For simplicity we add an extra starting node $h$ with empty bag and degree 1 to the tree decomposition and set $r=h$ initially. Note that an empty bag cannot be editable. Now, for all nodes there are three states - unseen, open, and closed. At start the node $h$ is open and other nodes are unseen. Let $W$ denote the bag of the node $r$. There are the following cases:

1. The node $r$ is open and has an unseen neighbor $s$ - Move $(s)$ and set $s$ open.

2. The node $r$ is open and has no unseen neighbors:

(a) It holds that $r=h$ - We are done, set $r$ as closed and return $T$.

(b) It holds that $|W| \leq w$ - Set $r$ as closed and Move $(s)$ to an open neighbor $s$ of $r$.

(c) It holds that $|W|=w+1$ and $W$ is not splittable - Return $t w(G)>k$.

(d) It holds that $|W|=w+1$ and $W$ is splittable - Split $W$, set the new bags as unseen, and move $r$ to a node that is adjacent to the new bags and is open.

Now, every time we use the Move operation in cases 1 and 2a-c we advance a state of a node and in case $2 \mathrm{~d}$ we create new nodes. Therefore, the number of moves is bounded by some constant times the number of nodes that appear in the tree decomposition over the course of the algorithm, which is $2^{O(w)} n$ by the potential function. To finish the argument it suffices to prove the correctness of the just described depth-first search implementation.

Lemma 4.1. The above described procedure maintains the invariant that the open nodes form a path from the starting node $h$ to $r$. Also, at the end of the procedure all nodes are closed.

Proof. Cases 1 and 2a-c clearly maintain the invariant. For $2 \mathrm{~d}$, the removed subtree contained $r$ but not $h$ because the bag of $h$ is empty, so the first node on the path from $r$ to $h$ that was not editable is the only node adjacent to the new nodes that is open. To see that at the end all nodes are closed note that we also maintain an invariant that if a node $i$ is closed and is in the path from a node $j$ to $h$ then $j$ is also closed.

\subsection{The Data Structure}

We explain how to implement the data structure. The data structure is essentially a dynamic programming table on the underlying tree decomposition $T$, directed towards the root node $r$. The 
main ideas are that moving $r$ to an adjacent node $s$ changes the dynamic programming tables of $r$ and $s$ only, and that we do not need to store any extra information on how the solution intersects with the query set because the query set is equal to the root bag. We note that all of the $2^{O(w)}$ factors in the running times of the data structure operations are $4^{w} w^{O(1)}$.

\subsubsection{Stored Information}

Let $i$ be a node of $T$ with a bag $B_{i}$, and $G\left[T_{i}\right]$ be the subgraph of $G$ induced by vertices in the bags of the rooted subtree of $T$ rooted at $i$. For each partition $\left(C_{1} \cap B_{i}, C_{2} \cap B_{i}, C_{3} \cap B_{i}, X \cap B_{i}\right)$ of $B_{i}$ and integer $0 \leq h \leq w$ we have a table entry $U[i]\left[\left(C_{1} \cap B_{i}, C_{2} \cap B_{i}, C_{3} \cap B_{i}, X \cap B_{i}\right)\right][h]$. This table entry stores $\perp$ if there is no partition $\left(C_{1}, C_{2}, C_{3}, X\right)$ of $V\left(G\left[T_{i}\right]\right)$ such that $|X|=h$ and there are no edges between $C_{1}, C_{2}, C_{3}$. If there is such a partition, then we store the minimum possible integer $d(X)$ over all such partitions, defined as $d(X)=\sum_{x \in X} d(x)$, where $d(x)=0$ if $x \in B_{i}$ and otherwise $d(x)$ is the distance in $T$ between $B_{i}$ and the closest descendant bag of $B_{i}$ that contains $x$. In particular if $B_{i}$ is the root bag then $d(X)$ is the function that is minimized on a minimum split.

\subsubsection{Transitions}

Let $B_{i}$ be a bag with at most 3 child bags $B_{a}, B_{b}, B_{c}$. We now describe how to compute in $2^{O(w)}$ time the table entries $U[i][\ldots][\ldots]$ given the table entries of $U[\{a, b, c\}][\ldots][\ldots]$.

First, we edit the stored distances in the entries $U[\{a, b, c\}][\ldots][\ldots]$ to correspond to distances from $B_{i}$. In particular, for an entry $U[j]\left[\left(C_{1} \cap B_{j}, C_{2} \cap B_{j}, C_{3} \cap B_{j}, X \cap B_{j}\right)\right][h] \neq \perp$ we increment the stored distance $d(X)$ by $h-\left|X \cap B_{j} \cap B_{i}\right|$. Then we do the transition by first decomposing it into $O(w)$ "nice" transitions of types introduce, forget, and join. In an introduce transition we have a bag $B_{i}$ with a single child bag $B_{j}$ with $B_{j} \subseteq B_{i}$ and $\left|B_{i} \backslash B_{j}\right|=1$, in a forget transition we have a bag $B_{i}$ with a single child bag $B_{j}$ with $B_{i} \subseteq B_{j}$ and $\left|B_{j} \backslash B_{i}\right|=1$, and in a join transition we have a bag $B_{i}$ with two child bags $B_{j}, B_{k}$ with $B_{j}=B_{k}=B_{i}$. The decomposition is done by first forgetting every vertex not in $B_{i}$, then introducing every vertex in $B_{i}$, and then joining.

The transitions that we have are standard and can be done as follows in time $2^{O(k)}$. We define $U[\ldots][\ldots][h]=\perp$ for all $h<0$ and for all $h>w$.

- Introduce: Let $\{v\}=B_{i} \backslash B_{j}$. We set $U[i]\left[\left(C_{1} \cap B_{i}, C_{2} \cap B_{i}, C_{3} \cap B_{i}, X \cap B_{i}\right)\right][h]=$ $U[j]\left[\left(C_{1} \cap B_{j}, C_{2} \cap B_{j}, C_{3} \cap B_{j}, X \cap B_{j}\right)\right][h-|\{v\} \cap X|]$ if there are no edges between $C_{1} \cap B_{i}$, $C_{2} \cap B_{i}, C_{3} \cap B_{i}$ and otherwise to $\perp$.

- Forget: Let $\{v\}=B_{j} \backslash B_{i}$. We set $U[i]\left[\left(C_{1} \cap B_{i}, C_{2} \cap B_{i}, C_{3} \cap B_{i}, X \cap B_{i}\right)\right][h]$ to be the minimum value over the values $U[j]\left[\left(C_{1} \cap B_{i} \cup\{v\}, C_{2} \cap B_{i}, C_{3} \cap B_{i}, X \cap B_{i}\right)\right][h], U[j]\left[\left(C_{1} \cap\right.\right.$ $\left.\left.B_{i}, C_{2} \cap B_{i} \cup\{v\}, C_{3} \cap B_{i}, X \cap B_{i}\right)\right][h], U[j]\left[\left(C_{1} \cap B_{i}, C_{2} \cap B_{i}, C_{3} \cap B_{i} \cup\{v\}, X \cap B_{i}\right)\right][h]$, and $U[j]\left[\left(C_{1} \cap B_{i}, C_{2} \cap B_{i}, C_{3} \cap B_{i}, X \cap B_{i} \cup\{v\}\right)\right][h]$, where $\perp$ is treated as a larger value than any integer.

- Join: Let $B_{j}, B_{k}$ be the child bags of $B_{i}$. We set $U[i]\left[\left(C_{1} \cap B_{i}, C_{2} \cap B_{i}, C_{3} \cap B_{i}, X \cap B_{i}\right)\right][h]=$ $\min _{h_{1}+h_{2}=h+\left|X \cap B_{i}\right|} U[j]\left[\left(C_{1} \cap B_{i}, C_{2} \cap B_{i}, C_{3} \cap B_{i}, X \cap B_{i}\right)\right]\left[h_{1}\right]+U[k]\left[\left(C_{1} \cap B_{i}, C_{2} \cap B_{i}, C_{3} \cap\right.\right.$ $\left.\left.B_{i}, X \cap B_{i}\right)\right]\left[h_{2}\right]$, where $\perp+n=\perp$ for any integer $n$. Note that we do not double count $d(x)$ for any $x \in X$ because if $x$ is in both subtrees of $j$ and $k$ then it is also in $B_{i}$ and therefore has $d(x)=0$. 


\subsubsection{Split Query}

Now the Split query on the node $r$ with bag $W$ amounts to iterating over all integers $0 \leq h \leq w$ and partitions $\left(C_{1} \cap W, C_{2} \cap W, C_{3} \cap W, X \cap W\right)$ such that $\left|\left(W \cap C_{i}\right)\right|+h<|W|$ for all $i$, and returning $\perp$ if the entries of all of them contain $\perp$ and otherwise returning $T$. Also the internal state of $r$ will be set to a pair $\left(\left(C_{1} \cap W, C_{2} \cap W, C_{3} \cap W, X \cap W\right), h\right)$ such that $U[r]\left[\left(C_{1} \cap W, C_{2} \cap W, C_{3} \cap W, X \cap W\right)\right][h]$ is not $\perp$, primarily minimizes $h$, and secondarily minimizes the stored integer $d(X)$. In particular, a split minimizing primarily $|X|$ and secondarily $d(X)$ is a minimum split. We also invalidate the internal states of other nodes by e.g. incrementing a global counter.

\subsubsection{Move Query}

Consider a move from a node $r$ to an adjacent node $s$.

First, if $r$ has an internal state and its children's internal states have been invalided we use the internal state $\left(\left(C_{1} \cap W, C_{2} \cap W, C_{3} \cap W, X \cap W\right), h\right)$ of $W$ to compute the corresponding internal states of its child nodes by implementing the dynamic programming transitions backwards. We also do the same for $s$ after $r$.

Then, when moving the root from a node $r$ to an adjacent node $s$ the only edge whose direction towards the root changes is the edge between $r$ and $s$. Therefore we first re-compute the table of $r$ and then the table of $s$.

\subsubsection{Initialization}

We initialize the tables with the already described transitions. For an empty subgraph $V\left(G\left[T_{i}\right]\right)=\emptyset$ we have $U[i][(\emptyset, \emptyset, \emptyset, \emptyset)][0]=0$ and $U[i][(\emptyset, \emptyset, \emptyset, \emptyset)][h]=\perp$ for $h \neq 0$.

\subsubsection{State Query}

With the move queries we have already pushed the internal state of the current node to be valid, so we just return it.

\subsubsection{Edit Query}

Consider an edit query that replaces a subtree $T_{1}$ with $T_{2}$, where $r \in T_{1}$. Because $r \in T_{1}$, all the dynamic programming tables are already oriented towards the subtree $T_{1}$. Therefore we just destroy the tables of $T_{1}$. We construct the new tables by inserting the nodes of $T_{2}$ one by one by applying $\left|T_{2}\right|$ transitions.

\section{Analysis of the $2^{O(k)}$ Factor in the Time Complexity}

In this section we briefly give an upper bound for the $2^{O(k)}$ factor in the time complexity of our algorithm, in order to support our claim that this factor in our algorithm is significantly smaller than in the algorithms of $\left[\mathrm{BDD}^{+} 16\right]$.

First, we note that the potential function can be optimized. In particular, a potential function of $\phi(T)=\sum_{i \in V(T)}\left(2\left[\left|B_{i}\right|=w+1\right]+1\right)\left|B_{i}\right| 3^{\left|B_{i}\right|}$, where $\left[\left|B_{i}\right|=w+1\right]$ denotes a value that is 1 if $\left|B_{i}\right|=w+1$ and 0 otherwise, also works in Lemma 3.3 and has an upper bound of $O\left(3^{w} w n\right)$, where $w$ is the width of $T$.

Second, we note that if the width $w$ of the given tree decomposition is at least $3 k+3$, then we can use 2-way splits instead of 3-way splits. 
Lemma 5.1. Let $G$ be a graph of treewidth $\leq k$. Any vertex set $W \subseteq V(G)$ of size $|W| \geq 3 k+4$ has a split of form $\left(C_{1}, C_{2}, \emptyset, X\right)$.

Proof. Again, as in Lemma 3.1, let $X$ be a balanced separator of $W$ of size $|X| \leq k+1$, and let us combine the two components $C_{i}$ of $G \backslash X$ with the smallest sizes of $C_{i} \cap W$ until we obtain a partition $\left(C_{1}, C_{2}, X\right)$ of $V(G)$. By considering the cases of whether there is a component $C_{i}$ with $\left|W \cap C_{i}\right| \geq|W| / 3$ or not, we notice that we will end up with $\left|W \cap C_{i}\right| \leq 2|W| / 3$ for both $i \in\{1,2\}$. Therefore $\left(C_{1}, C_{2}, \emptyset, X\right)$ is a split of $W$ because $\left|\left(W \cap C_{i}\right) \cup X\right| \leq 2|W| / 3+k+1<|W|$.

Now, if the width $w$ of the input tree decomposition is $w \geq 3 k+3$, we apply a version of the algorithm that only considers 2 -way splits, i.e., fixes $C_{3}=\emptyset$. This reduces the time complexity of the data structure operations from $4^{w} w^{O(1)}$ to $3^{w} w^{O(1)}$. In this case also the factor $3^{\left|B_{i}\right|}$ in the potential function can be replaced by a factor $2^{\left|B_{i}\right|}$. Therefore, in the case that $w \geq 3 k+3$, the total time complexity is $2^{w} 3^{w} w^{O(1)} n$, which by $w \leq 4 k+3$ is at most $1296^{k} k^{O(1)} n$. In the case that $w \leq 3 k+2$, the total time complexity is $3^{w} 4^{w} w^{O(1)} n \leq 1728^{k} k^{O(1)} n$.

\section{Conclusion}

We gave a $2^{O(k)} n$ time 2-approximation algorithm for treewidth. This is the first 2-approximation algorithm for treewidth that is faster than the known exact algorithms, and improves the best approximation ratio achieved in time $2^{O(k)} n$ from 5 to $2\left[\mathrm{BDD}^{+} 16\right]$.

Our algorithm improves upon the algorithm of Bodlaender et al. [BDD $\left.{ }^{+} 16\right]$ also in the running time dependency on $k$ hidden in the $2^{O(k)}$ notation. Bodlaender et al. do not include an analysis of this factor in their work, nor attempt to optimize this factor in any way, but we note that their algorithm makes use of dynamic programming with time complexity $\Omega\left(9^{w}\right)$ on a tree decomposition of width $w$, where an upper bound for $w$ is $30 k$, yielding a 29-digit number as the base of the exponent. While our algorithm constitutes progress in improving the dependency on $k$, the problem of finding a constant-factor treewidth approximation algorithm with running time $c^{k} n$, where the constant $c$ is small, remains open. Nevertheless, we believe that despite somewhat impractical worst-case bounds, our techniques are well applicable for practical implementations, and in fact, the MSVS heuristic proposed in [KBvH01] already resembles our algorithm on some aspects.

An interesting feature of our algorithm is that the only place where the approximation ratio 2 appears is in Lemma 3.1. In particular, bags of size less than $2 k+3$ can be splittable, and our algorithm could continue to split bags even after reaching a tree decomposition of width $2 k+1$. An interesting direction for future work would be to further analyze the cases where our algorithm actually gets stuck with a suboptimal tree decomposition, with the goal of either managing to avoid these cases by using additional techniques or finding graph classes where they do not exist. For this purpose, we note that the 3-way splits of our algorithm could well be generalized to $r$-way splits for any $r \geq 2$. Another natural direction for future work could be to extend our approach to approximating also other graph parameters that can be defined via tree decompositions.

\section{Acknowledgements}

I thank Otte Heinävaara, Mikko Koivisto, and Hans Bodlaender for helpful comments on these results and their presentation. 


\section{References}

[ACP87] Stefan Arnborg, Derek G. Corneil, and Andrzej Proskurowski. Complexity of finding embeddings in a k-tree. SIAM Journal on Algebraic Discrete Methods, 8(2):277-284, 1987. 1, 2

[Ami10] Eyal Amir. Approximation algorithms for treewidth. Algorithmica, 56(4):448-479, 2010. 1,2

[AP89] Stefan Arnborg and Andrzej Proskurowski. Linear time algorithms for NP-hard problems restricted to partial k-trees. Discrete Applied Mathematics, 23(1):11-24, 1989. 1

[BCKN15] Hans L. Bodlaender, Marek Cygan, Stefan Kratsch, and Jesper Nederlof. Deterministic single exponential time algorithms for connectivity problems parameterized by treewidth. Information and Computation, 243:86-111, 2015. 1

[BD02] Patrick Bellenbaum and Reinhard Diestel. Two short proofs concerning treedecompositions. Combinatorics, Probability and Computing, 11(6):541-547, 2002. 2, 5

[BDD $\left.{ }^{+} 13\right]$ Hans L. Bodlaender, Pål Grønås Drange, Markus S. Dregi, Fedor V. Fomin, Daniel Lokshtanov, and Michal Pilipczuk. An $\mathrm{O}\left(c^{k} n\right)$ 5-approximation algorithm for treewidth. In Proceedings of the 54th Annual IEEE Symposium on Foundations of Computer Science, FOCS 2013, pages 499-508. IEEE Computer Society, 2013. 1

[BDD $\left.{ }^{+} 16\right]$ Hans L. Bodlaender, Pål Grønås Drange, Markus S. Dregi, Fedor V. Fomin, Daniel Lokshtanov, and Michal Pilipczuk. A $\mathrm{c}^{\mathrm{k}} \mathrm{n}$ 5-approximation algorithm for treewidth. SIAM Journal on Computing, 45(2):317-378, 2016. 1, 2, 3, 4, 5, 11, 12

[BJT21] Hans L. Bodlaender, Lars Jaffke, and Jan Arne Telle. Typical sequences revisited computing width parameters of graphs. Theory of Computing Systems, pages 1-37, 2021. 2

[Bod88] Hans L. Bodlaender. Dynamic programming on graphs with bounded treewidth. In Timo Lepistö and Arto Salomaa, editors, Proceedings of the 15th International Colloquium on Automata, Languages and Programming, ICALP 1988, volume 317 of Lecture Notes in Computer Science, pages 105-118. Springer, 1988. 1

[Bod93] Hans L. Bodlaender. A linear time algorithm for finding tree-decompositions of small treewidth. In S. Rao Kosaraju, David S. Johnson, and Alok Aggarwal, editors, Proceedings of the 25th Annual ACM Symposium on Theory of Computing, STOC 1993, pages 226-234. ACM, 1993. 1

[Bod96] Hans L. Bodlaender. A linear-time algorithm for finding tree-decompositions of small treewidth. SIAM Journal on Computing, 25(6):1305-1317, 1996. 1, 2, 3, 4

$\left[\mathrm{CKL}^{+} 21\right]$ Marek Cygan, Pawel Komosa, Daniel Lokshtanov, Marcin Pilipczuk, Michal Pilipczuk, Saket Saurabh, and Magnus Wahlström. Randomized contractions meet lean decompositions. ACM Transactions on Algorithms, 17(1):6:1-6:30, 2021. 2 
[Cou90] Bruno Courcelle. The monadic second-order logic of graphs. I. Recognizable sets of finite graphs. Information and Computation, 85(1):12-75, 1990. 1

[EJT10] Michael Elberfeld, Andreas Jakoby, and Till Tantau. Logspace versions of the theorems of bodlaender and courcelle. In Proceedings of the 51th Annual IEEE Symposium on Foundations of Computer Science, FOCS 2010, pages 143-152. IEEE Computer Society, 2010. 2

[FHL08] Uriel Feige, MohammadTaghi Hajiaghayi, and James R. Lee. Improved approximation algorithms for minimum weight vertex separators. SIAM Journal on Computing, 38(2):629-657, 2008. 1, 2

[FLS $\left.{ }^{+} 18\right]$ Fedor V. Fomin, Daniel Lokshtanov, Saket Saurabh, Michal Pilipczuk, and Marcin Wrochna. Fully polynomial-time parameterized computations for graphs and matrices of low treewidth. ACM Transactions on Algorithms, 14(3):34:1-34:45, 2018. 1, 2

[FTV15] Fedor V. Fomin, Ioan Todinca, and Yngve Villanger. Large induced subgraphs via triangulations and CMSO. SIAM Journal on Computing, 44(1):54-87, 2015. 1

[GJNW21] Carla Groenland, Gwenaël Joret, Wojciech Nadara, and Bartosz Walczak. Approximating pathwidth for graphs of small treewidth. In Dániel Marx, editor, Proceedings of the 2021 ACM-SIAM Symposium on Discrete Algorithms, SODA 2021, pages 1965-1976. SIAM, 2021. 2

[HW17] Daniel J. Harvey and David R. Wood. Parameters tied to treewidth. Journal of Graph Theory, 84(4):364-385, 2017. 2

[KBvH01] Arie M. C. A. Koster, Hans L. Bodlaender, and Stan P. M. van Hoesel. Treewidth: Computational experiments. Electronic Notes in Discrete Mathematics, 8:54-57, 2001. 12

[Klo94] Ton Kloks. Treewidth, Computations and Approximations, volume 842 of Lecture Notes in Computer Science. Springer, 1994. 8

[Lag96] Jens Lagergren. Efficient parallel algorithms for graphs of bounded tree-width. Journal of Algorithms, 20(1):20-44, 1996. 1, 2

[LSS20] Daniel Lokshtanov, Saket Saurabh, and Vaishali Surianarayanan. A parameterized approximation scheme for min $k$-cut. In 61st IEEE Annual Symposium on Foundations of Computer Science, FOCS 2020, pages 798-809. IEEE, 2020. 2

[PR00] Ljubomir Perkovic and Bruce A. Reed. An improved algorithm for finding tree decompositions of small width. International Journal of Foundations of Computer Science, 11(3):365-371, 2000. 2

[Ree92] Bruce A. Reed. Finding approximate separators and computing tree width quickly. In S. Rao Kosaraju, Mike Fellows, Avi Wigderson, and John A. Ellis, editors, Proceedings of the 24th Annual ACM Symposium on Theory of Computing, STOC 1992, pages 221-228. ACM, 1992. 1, 2

[RS86] Neil Robertson and Paul D. Seymour. Graph minors. II. Algorithmic aspects of treewidth. Journal of Algorithms, 7(3):309-322, 1986. 4 
[RS95] Neil Robertson and Paul D. Seymour. Graph minors. XIII. The disjoint paths problem. Journal of Combinatorial Theory, Series B, 63(1):65-110, 1995. 1, 2

[WAPL14] Yu Wu, Per Austrin, Toniann Pitassi, and David Liu. Inapproximability of treewidth and related problems. Journal of Artificial Intelligence Research, 49:569-600, 2014. 2 